\title{
Unexpected changes in community size structure in a natural warming experiment
}

Eoin J. O’Gorman ${ }^{1, * \dagger}$, Lei Zhao ${ }^{2,1, \dagger}$, Doris E. Pichler ${ }^{3}$, Georgina Adams ${ }^{1}$, Nikolai Friberg ${ }^{4}$, Björn C. Rall ${ }^{5,6}$, Alex Seeney ${ }^{3}$, Huayong Zhang ${ }^{2}$, Daniel C. Reuman $^{7,8, *}$, and Guy Woodward ${ }^{1, *}$.

${ }^{1}$ Imperial College London, Silwood Park Campus, Buckhurst Road, Ascot, Berkshire SL5 7PY, UK.

${ }^{2}$ Research Center for Engineering Ecology and Nonlinear Science, North China Electric Power University, Beijing, 102206, China.

${ }^{3}$ School of Biological and Chemical Sciences, Queen Mary University of London, Mile End Road, London E1 4NS, UK.

${ }^{4}$ NIVA, Norwegian Institute for Water Research, Gaustadalléen 21, NO-0349 Oslo, Norway.

${ }^{5}$ German Centre for Integrative Biodiversity Research (iDiv) Halle-Jena-Leipzig, Deutscher Platz 5, 04103 Leipzig, Germany.

${ }^{6}$ Institute of Ecology, Friedrich Schiller University Jena, Dornburger Str. 159, 07743 Jena, Germany.

${ }^{7}$ University of Kansas, Department of Ecology and Evolutionary Biology and Kansas Biological Survey, 2041 Haworth Hall 1200 Sunnyside Avenue Lawrence, Kansas 66045, USA.

${ }^{8}$ Laboratory of Populations, Rockefeller University, New York, NY, 10065, USA.

Classification: Biological Sciences - Ecology Type of article: Letter

${ }^{\dagger}$ These authors contributed equally to this work

* Corresponding authors: Eoin O'Gorman (e.ogorman@imperial.ac.uk), Daniel Reuman (reuman@ku.edu), Guy Woodward (guy.woodward@imperial.ac.uk) 
1 Natural ecosystems typically consist of many small and few large organisms ${ }^{1-4}$. The

2 scaling of this negative relationship between body mass and abundance has important

3 implications for resource partitioning and energy usage ${ }^{5-7}$. Global warming over the

4 next century is predicted to favour smaller organisms ${ }^{8-12}$, producing steeper mass-

5 abundance scaling ${ }^{13}$ and a less efficient transfer of biomass through the food web ${ }^{5}$.

6 Here, we show that the opposite effect occurs in a natural warming experiment

7 involving 13 whole-stream ecosystems within the same catchment, which span a

8 temperature gradient of $5-25{ }^{\circ} \mathrm{C}$. We introduce a mechanistic model that shows how the

9 temperature dependence of basal resource carrying capacity can account for these

10 previously unexpected results. If nutrient supply increases with temperature to offset

11 the rising metabolic demand of primary producers, there will be sufficient resources to

12 sustain larger consumers at higher trophic levels. These new data and the model that

13 explains them highlight important exceptions to some commonly assumed "rules" about

14 responses to warming in natural ecosystems.

Body mass $(M)$ is a key determinant of many ecological phenomena ${ }^{6,7,14}$ (e.g. growth, metabolism, feeding) and its relationship with abundance $(N)$ at either the individual or

17 species level is well described by a simple power law, $N \propto M^{b}$ (hereafter " $M N$-scaling").

18 The exponent $b$ and its controlling factors have generated considerable interest in community ecology for decades ${ }^{4,6}$, with widespread recognition that $b$ is related to energy flow through food webs ${ }^{5-7}$. Many studies have found that $M N$-scaling is conserved in the face of

21 biodiversity loss or species turnover and so may be a relatively stable property of ecosystems ${ }^{1-3}$. Thus, a change in $M N$-scaling may highlight a fundamental disruption to the processes that govern energy flow through an ecosystem by environmental or anthropogenic stressors. For example, steepening of size-spectra (i.e. a more negative exponent $b$ ) following fisheries exploitation is indicative of widespread losses at higher trophic levels ${ }^{5,15}$. 
Key processes that could lead to altered $M N$-scaling include species extinctions or invasions, altered bottom-up or top-down control, changes in growth rate or reproductive output, and evolutionary adaptation to new environments. Population dynamical models predict that large organisms from higher trophic levels will go extinct first in warmer environments ${ }^{16,17}$ because there is less energy available to them ${ }^{14}$, with empirical support from microcosm experiments ${ }^{11,17}$. The theoretical basis for warming-induced changes in size structure at lower trophic levels is less well-developed ${ }^{18,19}$, but there is widespread evidence for an increased prevalence of smaller organisms with warming ${ }^{8-10}$, albeit with variability depending on the size-range and ecosystem considered ${ }^{10,12}$. Fewer large and more small organisms should result in steeper $M N$-scaling, as demonstrated in experimental ponds where warming favoured smaller phytoplankton and led to steeper size-spectra ${ }^{13}$.

We tested the generality of this predicted temperature effect on $M N$-scaling across 13 Icelandic streams that span a natural temperature gradient of $5-25{ }^{\circ} \mathrm{C}$ (Fig. 1a), but are otherwise very similar in their physical and chemical properties ${ }^{20-24}$. Natural experiments and space-for-time substitutions have some limitations (e.g. non-random allocation of temperature "treatments", no observation of the warming process but rather its end point; see Supplementary Methods for discussion of these limitations), however, the streams occur in the same catchment and thus are free of the usual confounding effects of biogeographical differences or other environmental gradients ${ }^{23,25}$. The constituent species are a subset of those commonly found in continental Europe and North America ${ }^{23}$, with compositional differences between the streams reflecting the local filtering of cold-stenotherms, warm-stenotherms, and eurytherms from the regional species pool $^{22}$. The streams are thus an invaluable natural experiment for improving our ecosystem-level understanding of warming impacts ${ }^{23,25}$. Individual organisms were measured and counted from every stream $(n=13,085$ individuals) to estimate the mean body mass and total abundance of each species (see 
51 Methods). There was an interactive effect of body mass and stream temperature on abundance, i.e. temperature altered $M N$-scaling (Table 1). Contrary to traditional theoretical predictions, the exponent $b$ became less negative as temperature increased (Fig. 1b,c; Fig. S1). This shallowing of $M N$-scaling was driven by differences across streams in two major trophic groups: primary producers and invertebrate consumers. Among the former, the abundance (Fig. 1d) and biomass (Fig. S2g) of diatoms decreased with temperature, contrary to the species shift hypothesis that warming should increase the abundance of small species ${ }^{8}$. Manipulative experiments suggest that this may be due to greater top-down control by grazers $^{22}$. The mean body mass of invertebrates increased with temperature (Fig. 1e), in diametric opposition to the community body size shift hypothesis ${ }^{8}$. This was largely driven by compositional changes, with bigger species (such as the snail, Radix balthica) only occurring in warmer streams and dominating those communities ${ }^{21-23}$.

The temperature effect on $M N$-scaling still held after quantifying only the mean body mass and total abundance of the major trophic groups (Table S1; Fig. S3), including additional data for cryptic biota that are typically overlooked and rarely quantified in freshwater studies (Table S2; Fig. S4), and excluding data for the apex fish predator, brown trout (Salmo trutta), which only occurs in the warmer streams (Tables 1, S1, and S2; Fig. 1c). Our findings were also robust to various methodological approaches, including different methods of averaging (Table S3; Fig. S5), regression model selection (Table S4; Fig. S6), and binning by individual size data (Table S5; Fig. S7). We focused on diatoms as the key primary producers in the system, but analysis of total chlorophyll (including diatoms, cyanobacteria, and green algae) did not alter our conclusions about the effect of temperature on the biomass of primary producers (Fig. S8).

To explain these apparent contradictions with established theory, we hypothesised that the equilibrium biomass of basal resources in the absence of consumption (i.e. the carrying 
capacity, $K$ ) could play a critical role. For algae, $K$ is determined by the balance between nutrient supply and demand ${ }^{14,26}$. Our study streams are co-limited by nitrogen and phosphorus, with nitrogen being the key limiting nutrient ${ }^{21}$, i.e. the demand for nutrients will predominantly be met by the nitrogen and phosphorus cycles and input of these elements from groundwater or terrestrial sources. For autotrophs, the metabolic demand for nutrients is equal to the rate of photosynthesis ${ }^{14}$. To assess the upper and lower bounds of what is feasible in our system, we tested two extreme scenarios ${ }^{26}$ for the temperature dependence of $K:(i)$ if nutrient supply is constant, $K$ should decrease with increasing temperature to exactly balance the increasing photosynthetic rate, i.e. with an activation energy, $E_{K}$, of -0.70 to -0.96 , representing the inverse of the $95 \%$ confidence intervals (CI) of published temperature dependencies for photosynthesis in aquatic microalgae (see Supplementary Methods and Table S6); and (ii) if nutrient supply increases with temperature to a level that exactly matches the photosynthetic rate, $K$ should be independent of temperature, i.e. $E_{K}=0$.

Three lines of evidence suggest that the rate of nutrient supply increases with temperature in our system: $(i)$ nitrogen fixation increases dramatically with temperature ${ }^{27}$; (ii) water-column concentrations of nutrients are not depleted with temperature ${ }^{20-24}$, as would be expected due to the rising metabolic demand of primary producers if nutrient supply were constant; and (iii) the body mass of diatoms does not decrease with temperature ${ }^{20}$ (Fig. S2d), as would be expected if competition for nutrients were strong ${ }^{19}$. Additionally, headwater streams are among the most metabolically active freshwaters due to regular replenishment of nutrients from surface to sub-surface exchanges ${ }^{28}$. Many headwater streams also exhibit biogeochemical steady state along their entire length, with nutrient inputs balancing outputs and nutrient concentrations similar to those of soil and groundwater ${ }^{29}$. Thus, we hypothesise that the temperature dependence of $K$ cannot be entirely driven by the photosynthetic rate in our system, and that $E_{K}$ determines $M N$-scaling. 
We tested this hypothesis using a bioenergetic population dynamical model, which contains free parameters for the growth rate and $K$ of primary producers, the metabolic and attack rates of invertebrates and fish, and estimates of the measurement error for the biomass of each trophic group (see Methods). We determined the combination of parameters that best fitted our empirical data using maximum likelihood. The optimum model explained 32\%, $84 \%$, and $97 \%$ of the variation across streams in the empirical biomass of diatoms, invertebrates, and fish, respectively (Fig. 2a), and had estimates for most parameters that overlapped with published values from other freshwater ecosystems (Table S7). The value of $E_{K}$ that best described our data was -0.30 , with $95 \%$ CI of -0.47 and 0.20 . This range does not overlap with the $95 \%$ CI of $E_{K}$ predicted for a constant nutrient supply (-0.70 to -0.96 ; Table S6), so we reject the null hypothesis that $E_{K}$ is entirely driven by the photosynthetic rate. We carried out a sensitivity analysis to determine the effect of $E_{K}$ on $M N$-scaling. Here, we fixed all the parameter values from our best-fitting model except for $E_{K}$, which we varied

114 from -1 to 0.5 . The predicted steepening of $M N$-scaling with increasing temperature only 115 occurred for $E_{K}<-0.33$, with the observed shallowing of $M N$-scaling found when $E_{K}>-0.33$

116 (Fig. 2b). This suggests that $E_{K}$ plays a critical role in determining the effect of temperature on $M N$-scaling, i.e. the rate at which nutrient supply increases with temperature can offset the increasing photosynthetic rate, supporting a higher than expected $K$ of basal resources and thus larger biomass of consumers.

The $5-25{ }^{\circ} \mathrm{C}$ temperature gradient of our streams is well within the thermal limits for survival of brown trout ${ }^{30}$, so it is surprising that this fish species was only found $>15{ }^{\circ} \mathrm{C}$ (Fig. 2a), with similar results documented in a $>5$ month fish tagging study from the system ${ }^{31}$. Our model can also help to understand these seemingly unexpected results. Resource production

124 is converted to consumer production more efficiently as stream temperature increases ${ }^{31}$ (Fig.

125 S9). This may be driven by increasing dominance of $R$. balthica, which is the largest 
126 herbivore in the system ${ }^{21-23}$. This highly efficient snail exerts stronger grazing pressure with

127 increasing temperature ${ }^{22}$ and thus may be a key conduit of energy flow to the fish. Mass-

128 specific metabolic requirements are lower for larger organisms ${ }^{14}$, so their population biomass

129 should be higher, given the same amount of resources. Thus, we also hypothesised that the

130 previously unexpected increase in the body mass of invertebrates with temperature (Fig. 1e) supported greater fish biomass in the warmer streams. We fixed all parameters at values from our best-fitting model, except for the temperature dependence of invertebrate body mass $\left(E_{M 2}\right)$ and, for each value of $E_{M 2}$, determined the minimum model-predicted temperature at

134 which fish were present in a stream. We found that the positive relationship between 135 invertebrate body mass and temperature was critically important and that fish would not be supported if the relationship were negative, as predicted by temperature-size rules (Fig. 2c).

We have shown that the temperature dependencies of $K$ and consumer body mass can modulate how warming affects energy flow through food webs in a previously unexpected manner. Thus, if resource production is sufficient in warmer environments, larger consumers may be sustained by a lower standing stock (i.e. abundance) of resources (Fig. 1c-e). While many of the studies investigating effects of temperature on the size structure of aquatic communities have focused on the lowest trophic level (e.g. microalgae) $)^{9,20}$, our research highlights the potential for warming to alter the size distribution of unicells, ectothermic invertebrates, and vertebrates across $>12$ orders of magnitude in body mass, and hence the

145 flow of energy through the entire ecosystem. Larger apex predators have the potential to exert 146 stronger top-down control, with effects that can cascade down to the lower trophic levels ${ }^{32}$, 147 but manipulative experiments would be needed to fully disentangle the direct effects of 148 temperature from indirect effects due to stronger feeding at the top of the food web.

149 It is important to consider the context of our findings before attempting to generalise 150 them to future impacts of climate change. The streams are quite species-poor, although the 
151 key taxa are common throughout Europe and/or North America ${ }^{23}$, so the results may be most relevant for Northern Hemisphere upland and/or headwater ecosystems with similarly low

153 biodiversity. Our temperature gradient is substantial, with a range of $20{ }^{\circ} \mathrm{C}$, which is more 154 than twice the projected warming for tundra regions in the $21^{\text {st }}$ century ${ }^{33}$. Nonetheless, the 155 warmest stream is within the upper thermal tolerance of most freshwater invertebrate taxa ${ }^{34}$. 156 As such, our results may be most relevant for ecosystems where constituent organisms are 157 well below their thermal limits, e.g. at cool, high latitudes, where other exceptions to 158 temperature-size rules have been identified ${ }^{10}$, rather than in the tropics or warm temperate 159 regions. For example, the thermal optimum for growth in brown trout is $11-19{ }^{\circ} \mathrm{C}$ (depending 160 on resource quality ${ }^{30}$ ) and so there is scope for improved performance over part of the 161 temperature range studied here ${ }^{31}$. The low productivity and nutrient-poor status of our 162 streams ${ }^{21,24}$ may also magnify the potential for increasing nutrient supply to offset higher metabolic demands at warmer temperatures. Nevertheless, our results contribute to a more general understanding of how warming could alter ecological communities because they suggest that changes in biomass at different trophic levels will depend on how the $K$ of primary producers is affected by temperature, and this is an insight that can be tested broadly. Our study system offers a powerful space-for-time substitution for warming impacts on natural communities, but also has limitations. Results from headwater streams may not scale up to larger ecosystems such as rivers, even though $M N$-scaling is consistently present across

170 a broad range of ecosystems and common underlying mechanisms have been proposed ${ }^{1,2,4,7}$

171 Whilst we avoided biogeographical gradients that confound some studies, the close proximity 172 of our streams could make it easier for organisms to disperse from the regional species pool to their optimum temperature than would be possible under a warming climate. Adaptation to warmer temperatures over many years of geothermal heating in the region may also produce

175 different organismal responses relative to rapid climate change ${ }^{25}$. Nevertheless, a recent 
whole-stream warming experiment from the system has revealed that changes in populations along the stream temperature gradient are similar to actual warming of a stream ${ }^{35}$.

Our results show that warming effects on $M N$-scaling can hinge crucially on the temperature dependence of $K$, mediated through nutrient dynamics, at least in ecosystems with high production rates and strong trophic linkages. We need a broader understanding of how $K$ depends on temperature in a range of environments (e.g. standing and flowing freshwaters, and marine and terrestrial ecosystems) to test the generality of our results further. Our data indicate that temperature-size rules, widely appreciated for their ubiquity ${ }^{8-10}$, do not apply universally in natural communities, with important implications for the higher trophic levels. Our results improve our understanding of the contingencies in temperature effects on natural ecosystems, which should enhance our ability to predict the ecological consequences of future climate change.

\section{Acknowledgements:}

We thank Julia Reiss for meiofauna and protist data, Nicola Craig for laboratory work, Aristides Moustakas for advice on data analysis, Gísli Már Gíslason and Jón S. Ólafsson for providing research support and facilities, and Gabriel Yvon-Durocher, Samraat Pawar, Mark Trimmer, and Becca Kordas for helpful comments on earlier drafts. We acknowledge funding from NERC (NE/I009280/2, NE/F013124/1, NE/L011840/1, NE/M020843/1), the Royal Society (RG140601), the British Ecological Society (4009-4884), the National Special Water Program (No. 2009ZX07210-009), the China Scholarship Council (No. 201206730022), the Department of Environmental Protection of Shandong Province (SDHBPJ-ZB-08), the German Research Foundation (FZT 118), the James S. McDonnell Foundation, and NSF (1442595). 
GW, NF, and DCR were responsible for funding application, research design, and planning. EOG, DEP, GA, and AS collected the data. EOG, BCR, and LZ analysed the data. LZ, DCR, and HZ did the modelling. All authors wrote the paper.

\section{References:}

2071 Jonsson, T., Cohen, J. E. \& Carpenter, S. R. Food webs, body size, and species 208 abundance in ecological community description. Advances in Ecological Research 36, $209 \quad 1-84(2005)$.

2102 Marquet, P. A., Navarrete, S. A. \& Castilla, J. C. Scaling population-density to body 211 size in rocky intertidal communities. Science 250, 1125-1127 (1990).

2123 O'Gorman, E. J. \& Emmerson, M. C. Body mass-abundance relationships are robust 213 to cascading effects in marine food webs. Oikos 120, 520-528 (2011).

2144 Reuman, D. C., Mulder, C., Raffaelli, D. \& Cohen, J. E. Three allometric relations of 215 population density to body mass: theoretical integration and empirical tests in 149 216 food webs. Ecology Letters 11, 1216-1228 (2008).

2175 Jennings, S. \& Blanchard, J. L. Fish abundance with no fishing: predictions based on 218 macroecological theory. Journal of Animal Ecology 73, 632-642 (2004).

2196 White, E. P., Ernest, S. K. M., Kerkhoff, A. J. \& Enquist, B. J. Relationships between 220 body size and abundance in ecology. Trends in Ecology \& Evolution 22, 323-330 $221 \quad$ (2007).

2227 Woodward, G. et al. Body size in ecological networks. Trends in Ecology \& Evolution 20, 402-409 (2005). 
2248 Daufresne, M., Lengfellner, K. \& Sommer, U. Global warming benefits the small in aquatic ecosystems. Proceedings of the National Academy of Sciences of the United States of America 106, 12788-12793 (2009).

2279 Moran, X. A. G., Lopez-Urrutia, A., Calvo-Diaz, A. \& Li, W. K. W. Increasing 228 importance of small phytoplankton in a warmer ocean. Global Change Biology 16, 1137-1144 (2010).

23010 Sheridan, J. A. \& Bickford, D. Shrinking body size as an ecological response to 231 climate change. Nature Climate Change 1, 401-406 (2011).

23211 Petchey, O. L., McPhearson, P. T., Casey, T. M. \& Morin, P. J. Environmental 233 warming alters food-web structure and ecosystem function. Nature 402, 69-72 (1999).

23412 Gardner, J. L., Peters, A., Kearney, M. R., Joseph, L. \& Heinsohn, R. Declining body 235 size: a third universal response to warming? Trends in Ecology \& Evolution 26, 285$291(2011)$.

23713 Yvon-Durocher, G., Montoya, J. M., Trimmer, M. \& Woodward, G. Warming alters 238 the size spectrum and shifts the distribution of biomass in freshwater ecosystems. 239 Global Change Biology 17, 1681-1694 (2011).

24014 Brown, J. H., Gillooly, J. F., Allen, A. P., Savage, V. M. \& West, G. B. Toward a 241 metabolic theory of ecology. Ecology 85, 1771-1789 (2004).

24215 Rice, J. \& Gislason, H. Patterns of change in the size spectra of numbers and diversity 243 of the North Sea fish assemblage, as reflected in surveys and models. ICES Journal of $244 \quad$ Marine Science 53, 1214-1225 (1996).

24516 Binzer, A., Guill, C., Brose, U. \& Rall, B. C. The dynamics of food chains under 246 climate change and nutrient enrichment. Philosophical Transactions of the Royal 247 Society of London B: Biological Sciences 367, 2935-2944 (2012). 
24817 Fussmann, K. E., Schwarzmüller, F., Brose, U., Jousset, A. \& Rall, B. C. Ecological 249 stability in response to warming. Nature Climate Change 4, 206-210 (2014).

25018 DeLong, J. P. Experimental demonstration of a 'rate-size'trade-off governing body 251 size optimization. Evolutionary Ecology Research 14, 343-352 (2012).

25219 Reuman, D. C., Holt, R. D. \& Yvon-Durocher, G. A metabolic perspective on 253 competition and body size reductions with warming. Journal of Animal Ecology 83, 59-69 (2014).

20 Adams, G. et al. Diatoms can be an important exception to temperature-size rules at species and community levels of organization. Global Change Biology 19, 3540-3552 (2013).

21 Friberg, N. et al. Relationships between structure and function in streams contrasting in temperature. Freshwater Biology 54, 2051-2068 (2009).

22 O'Gorman, E. J. et al. Impacts of warming on the structure and function of aquatic communities: individual- to ecosystem-level responses. Advances in Ecological Research 47, 81-176 (2012).

23 Woodward, G. et al. Sentinel systems on the razor's edge: effects of warming on Arctic geothermal stream ecosystems. Global Change Biology 16, 1979-1991 (2010).

24 Demars, B. O. L. et al. Temperature and the metabolic balance of streams. Freshwater Biology 56, 1106-1121 (2011).

26725 O'Gorman, E. J. et al. Climate change and geothermal ecosystems: natural laboratories, sentinel systems, and future refugia. Global Change Biology 20, 32913299 (2014).

26 Gilbert, B. et al. A bioenergetic framework for the temperature dependence of trophic interactions. Ecology Letters 17, 902-914 (2014). 
27227 Welter, J. R. et al. Does N2-fixation amplify the temperature dependence of 273 ecosystem metabolism? Ecology 96, 603-610 (2015).

27428 Battin, T. J. et al. Biophysical controls on organic carbon fluxes in fluvial networks. $275 \quad$ Nature Geosci 1, 95-100 (2008).

27629 Brookshire, E., Valett, H. \& Gerber, S. Maintenance of terrestrial nutrient loss 277 signatures during in-stream transport. Ecology 90, 293-299 (2009).

27830 Elliott, J. \& Elliott, J. Temperature requirements of Atlantic salmon Salmo salar, brown trout Salmo trutta and Arctic charr Salvelinus alpinus: predicting the effects of climate change. Journal of Fish Biology 77, 1793-1817 (2010).

$28131 \quad$ O'Gorman, E. J. et al. Temperature effects on fish production across a natural thermal gradient. Global Change Biology 22, 3206-3220 (2016).

28332 Kratina, P., Greig, H. S., Thompson, P. L., Carvalho-Pereira, T. S. \& Shurin, J. B. 284 Warming modifies trophic cascades and eutrophication in experimental freshwater 285 communities. Ecology 93, 1421-1430 (2012).

28633 IPCC. Working Group I contribution to the IPCC fifth assessment report. Climate 287 change 2013: the physical sciences basis summary for policymakers. 36 (Cambridge $288 \quad$ University Press, 2013).

28934 Stewart, B. A., Close, P. G., Cook, P. A. \& Davies, P. M. Upper thermal tolerances of 290 key taxonomic groups of stream invertebrates. Hydrobiologia 718, 131-140 (2013).

29135 Nelson, D. et al. Experimental whole-stream warming alters community size $292 \quad$ structure. Global Change Biology (2016). 
294 Table 1. Statistical output from linear mixed effects (LME) models. Ln-species

295 abundance $\left(\mathrm{m}^{-2}\right)$ was the dependent variable, ln-mean species body mass $(\mathrm{mg})$ and stream

296 temperature $\left({ }^{\circ} \mathrm{C}\right)$ were fixed effects, and species identity was a random effect. Data were for

29713 streams in August 2008 including diatoms, macroinvertebrates, and fish. Summary tables

298 for analyses (a) including and (b) excluding the apex fish predator are presented, with model-

299 predicted values, their standard errors (SE), degrees of freedom (DF), $t$-values, and $p$-values.

\begin{tabular}{llllll}
\hline (a) LME including fish & Value & SE & DF & $\boldsymbol{t}$-value & $\boldsymbol{p}$-value \\
\hline (Intercept) & 2.7966 & 0.3747 & 527 & 7.463 & $<0.0001$ \\
mass & -0.7228 & 0.0277 & 527 & -26.080 & $<0.0001$ \\
temperature & 0.3814 & 0.2380 & 527 & 1.603 & 0.1096 \\
mass $\times$ temperature & 0.0591 & 0.0184 & 527 & 3.204 & 0.0014
\end{tabular}

\begin{tabular}{llllll}
\hline (b) LME excluding fish & Value & SE & DF & $\boldsymbol{t}$-value & $\boldsymbol{p}$-value \\
\hline (Intercept) & 2.8084 & 0.3996 & 521 & 7.027 & $<0.0001$ \\
mass & -0.7221 & 0.0290 & 521 & -24.882 & $<0.0001$ \\
temperature & 0.4050 & 0.2510 & 521 & 1.614 & 0.1072 \\
mass $\times$ temperature & 0.0611 & 0.0194 & 521 & 3.153 & 0.0017
\end{tabular}


302 Fig. 1. Map of the geothermal streams and effects of temperature on mass-abundance

303 (MN) scaling. a, mean daytime temperature in August 2008 for 13 geothermally heated streams in Hengill, Iceland. b, predicted and observed effects of increasing temperature on $M N$-scaling: more small organisms, fewer large organisms, and/or decreasing body size are predicted in warmer environments, leading to a steeper $M N$ slope, but the opposite occurs in the Hengill streams. The dashed blue line is the $M N$ relationship in the coldest stream, to act as a reference point for the warmest stream. c, the slope of the $M N$ relationship, $b$, becomes shallower with increasing stream temperature. The solid and dashed lines are the results of the best-fitting models from analyses including and excluding fish, respectively (Table 1).

311 The circles and crosses are the $M N$ slopes for each stream from analyses including and excluding fish, respectively. d, ln-abundance of diatoms decreases with temperature $(y=$ $\left.-0.740 x+19.46, F_{1,11}=20.18, p<0.001, r^{2}=0.62\right)$. e, ln-body mass of invertebrates increases with temperature $\left(y=0.757 x-2.032 F_{1,11}=30.25, p<0.001, r^{2}=0.71\right)$. ecosystems. a, the best-fitting model (solid lines) closely approximates the empirical biomass of the three major trophic groups in the Hengill streams (circles). b, influence of the activation energy of carrying capacity, $E_{K}$, on the slope of the $M N$ relationship, $b$. A negative rate of change of $b$ with respect to temperature $\left({ }^{\circ} \mathrm{C}^{-1}\right)$ indicates a steepening of $M N$-scaling with warming as predicted by metabolic theory; this occurs for most negative values of $E_{K}$ (e.g. bottom-right inset). A positive rate of change of $b$ indicates a shallowing of $M N$-scaling, as observed in the empirical data; this only occurs for $E_{K}>-0.33$ (e.g. top-left inset). Circles indicate simulation results and solid lines are the fitted linear regressions. c, influence of the 
326 are present in a stream, $T_{m}$. The solid grey line indicates $E_{M 2}$ below which fish cannot invade 327 the system $\left(E_{M 2}=0.744\right)$; the empirical value of $E_{M 2}$ is only marginally higher than this $\left(E_{M 2}\right.$ $328=0.757)$. Values of $E_{M 2}$ beyond the dashed grey line are too large to be representative of the 329 data $\left(E_{M 2}=1.925\right)$. 
Fig. 1
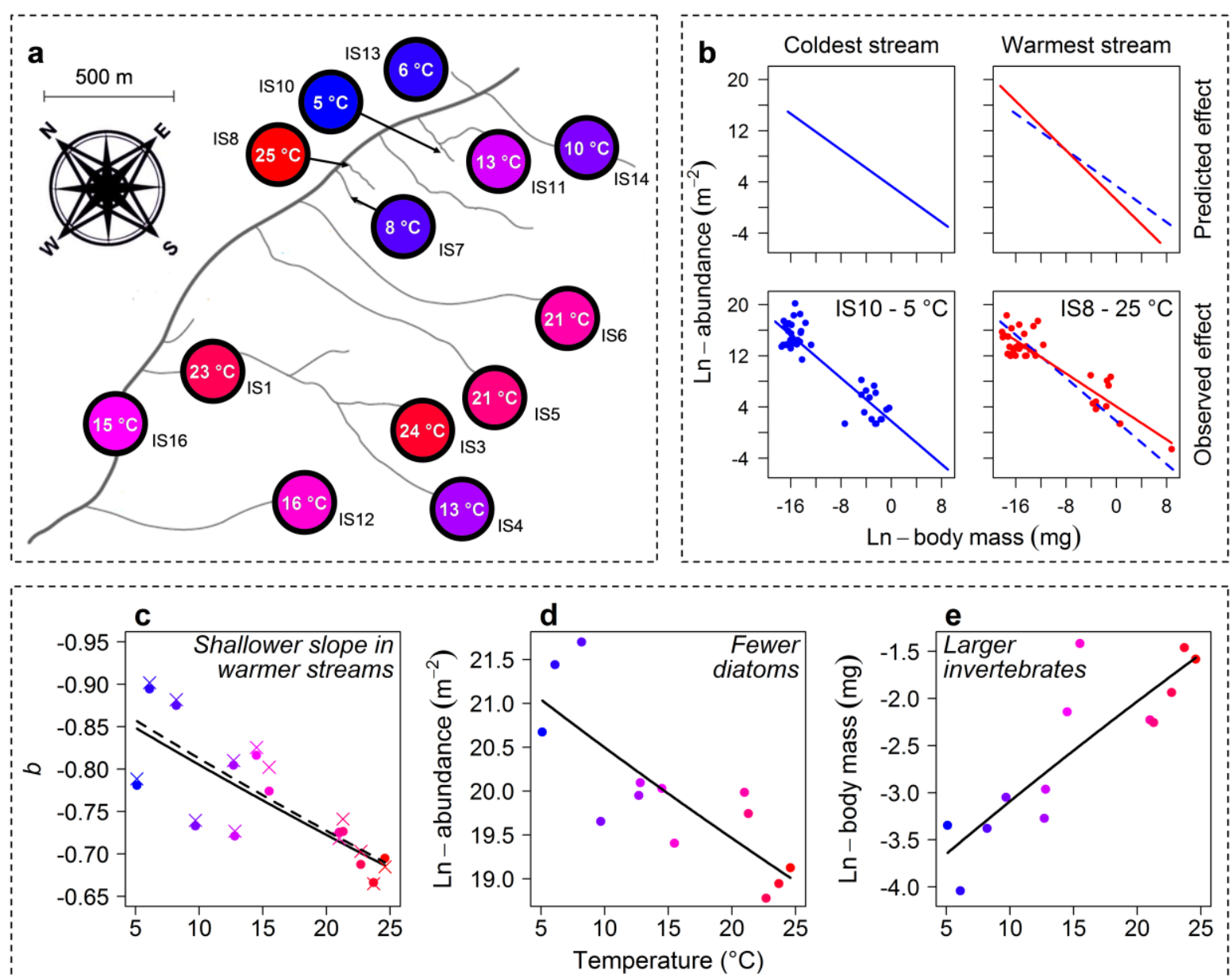
333 Fig. 2
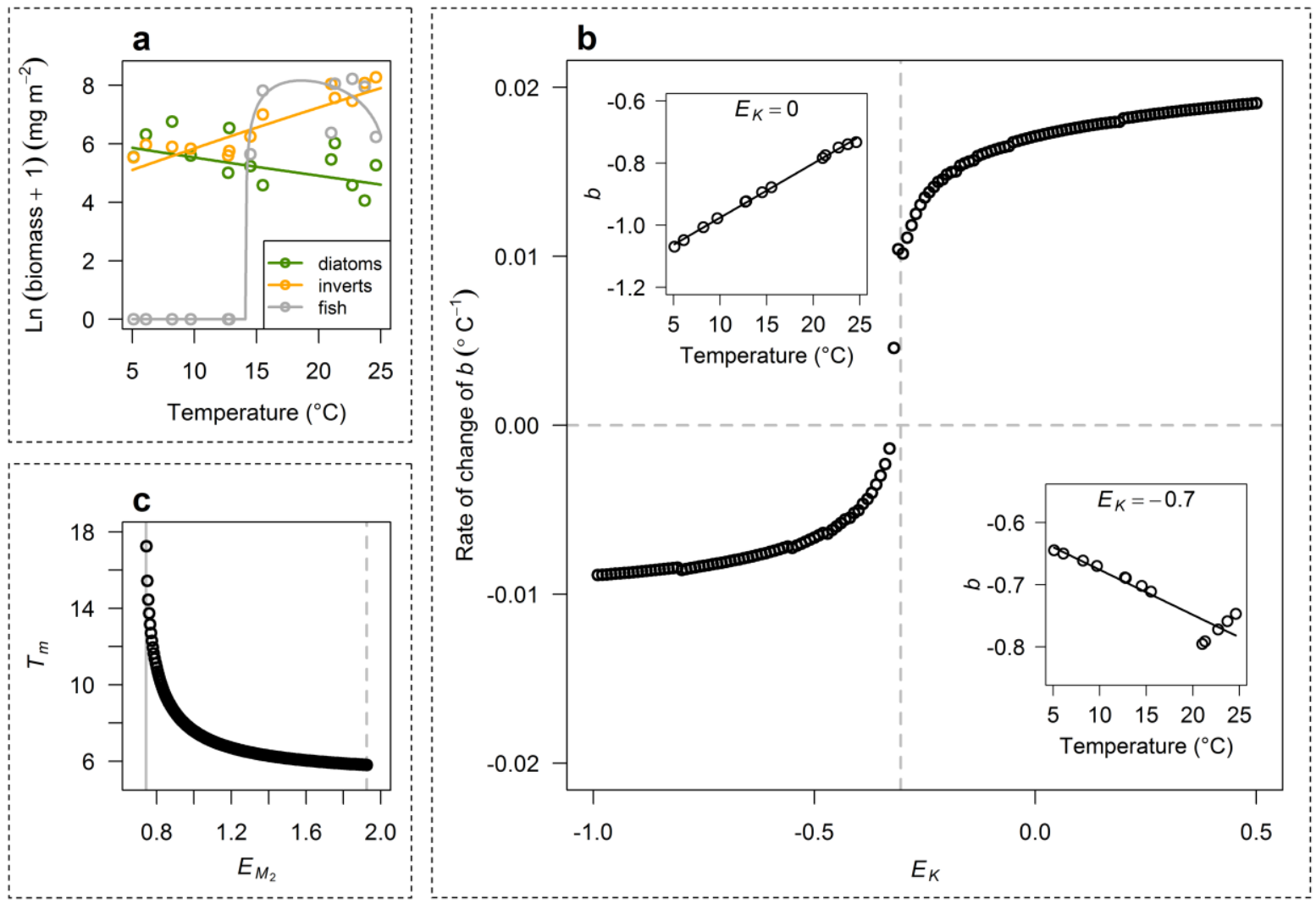

334

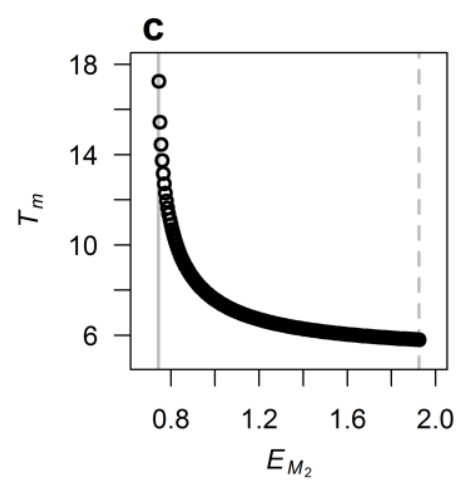




\section{Methods}

336

337

338

\section{Study site}

Fieldwork was performed in August 2008 in the Hengill geothermal valley, Iceland (N $64^{\circ} 03$; W $21^{\circ} 18$ ), which has been intensively studied over the past decade $20-24,31,35-37$. We focused on 13 streams that occur within $1.5 \mathrm{~km}$ of each other and spanned a temperature gradient of $5-25{ }^{\circ} \mathrm{C}$, which were also the minimum and maximum temperatures during the sampling period (see Table S8 and Supplementary Methods for more details). Note that most of the streams freeze over for part of the winter (Table S8), including several streams where fish are found (e.g. IS1, 3, and 12). There are also some streams that do not freeze which do not contain fish (e.g. IS13), suggesting that trout populations are not solely determined by winter freezing and are most likely sustained through interconnectivity with the main river ${ }^{31}$. Temperature differences between streams are due to groundwater that absorbs heat from the underlying bedrock, rather than direct upwelling of geothermal water and gases ${ }^{38}$. Thus, the streams have very similar water chemistry, with no confounding effects of temperature on $\mathrm{pH}$, derivatives of nitrogen and phosphorus, and a wide range of other minerals and nutrients ${ }^{20-24}$. The streams are also very similar in their physical characteristics ${ }^{21,24}$ and occur in a pristine mountain landscape, with no nutrient input or pollution from agriculture or industry. There are no trees or shrubs in the region, thus minimal coarse allochthonous input. The soil system exhibits a similar temperature gradient to the streams due to geothermal heating ${ }^{31}$, thus nutrient inputs from the soil should not be decoupled from temperature effects on nutrient dynamics in the streams. The only other external influence on the streams may come from rare occurrences of terrestrial predators, such as the golden plover and Arctic fox, and grazing by sheep. The streams are thus an ideal natural experiment for studying the effects of warmer temperatures on the structure of freshwater communities ${ }^{25}$ (but see Supplementary Methods for the strengths and weaknesses of natural experiments). 
Diatom abundance and body mass estimation

Diatoms were collected from three stone scrapes per stream (noting the area of each stone) and preserved in Lugol's solution. Diatom frustules were cleared of organic matter with nitric acid, dried, and mounted on slides with naphrax. Abundances were estimated by counting the number of individuals of each species along a $15 \times 0.1 \mathrm{~mm}$ transect of each slide, ensuring a transect contained at least 300 individuals. The number of stone scrapes, sample dilution, and transect and stone area were all used to calculate the abundance of each species $\left(\mathrm{m}^{-2}\right)$. Photographs of diatoms were taken with a Nikon Digital Sight DS-5M camera mounted on a Nikon Eclipse 50i microscope, or a high resolution digital SLR camera mounted on an Olympus $\mathrm{BH} 2$ microscope, at 1,000× magnification. Two linear dimensions were measured in Image $\mathrm{J}^{39}$ for at least ten individuals (where available) of every diatom species in every stream, i.e. valve length and valve width in microns ( $n=9,011$ individuals from 69 different taxa). Every diatom species was assigned a shape corresponding to established methodologies ${ }^{22,24}$ (Table S9). Cell biovolume $\left(\mu \mathrm{m}^{3}\right)$ was calculated according to associated biovolume formulae ${ }^{40}$. Cell carbon content was estimated from published cell volume to cell carbon relationships ${ }^{41}$ and converted to dry mass $(\mathrm{mg})$ assuming an average carbon by dry weight content of $19 \%$ per cell ${ }^{42}$.

\section{Macroinvertebrate abundance and body mass estimation}

380 Macroinvertebrates were collected by taking five Surber samples $(25 \times 20 \mathrm{~cm}$ quadrat; $200 \mu \mathrm{m}$ mesh) per stream and preserving them in $70 \%$ ethanol. The abundance of every invertebrate species was averaged across the five Surber samples and scaled by quadrat area $\left(\mathrm{m}^{-2}\right)$. Photographs of every invertebrate individual identified were taken with a Nikon Digital 


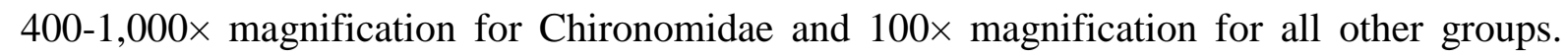

386 One linear dimension was measured in Image $\mathrm{J}^{39}$ for at least ten individuals (where available)

387 of every invertebrate species in every stream ( $n=4,121$ individuals from 42 different taxa).

388 Published length-weight relationships were used to estimate dry body mass (mg) from the

389 linear measurements (Table S10).

390

Fish abundance and body mass estimation

392

Only one fish species is found in the system: the brown trout, Salmo trutta. Population abundances $\left(\mathrm{m}^{-2}\right)$ of this species were characterised using three-run depletion electrofishing of a $50 \mathrm{~m}$ reach within a stream, or the entire stream if less than $50 \mathrm{~m}$ in length ${ }^{43}$.

395 Electrofishing of the entire catchment was carried out over a two day period. Body mass measurements of every fish $(n=53$ individuals) were made on a portable mass balance

(Ohaus Scout Pro Portable, $400 \mathrm{~g}$ capacity, $0.01 \mathrm{~g}$ accuracy). Dry mass (mg) of trout was calculated according to a wet weight to dry weight relationship established from 39 individuals of $S$. trutta $\left(y=1.088 x-0.878, F_{1,37}=1,201, p<0.0001, r^{2}=0.97\right)$. This fish species is orders of magnitude bigger than any other species in the streams and is thus the apex predator whenever it occurs. See Supplementary Methods for quantification of other trophic groups, including cryptic biota (meiofauna, ciliates, and flagellates) and unicellular algae other than diatoms (microscopic green algae and cyanobacteria).

Empirical exploration of $M N$-scaling

406

Population abundance should follow a power law with mean body mass ${ }^{6}$ and an 407 exponential relationship with temperature ${ }^{14}$ as follows:

$408 \quad N=a_{N} M^{b_{N}} e^{E_{N} T_{a r r}}$, 
409 Here, $N$ is total species abundance $\left(\mathrm{m}^{-2}\right), a_{N}$ is a constant, $b_{N}$ is the allometric exponent, $M$ is 410 mean species body mass $(\mathrm{mg}), E_{N}$ is the activation energy $(\mathrm{eV})$, and $T_{\text {arr }}$ is the standardised

411 Arrhenius temperature:

$412 T_{\text {arr }}=\frac{T-T_{0}}{k T T_{0}}$,

413 where $T$ is the absolute stream temperature $(\mathrm{K}), T_{0}$ is an arbitrary reference temperature $414(293.15 \mathrm{~K})$, and $k$ is the Boltzmann constant $\left(8.618 \times 10^{-5} \mathrm{eV} \mathrm{K}^{-1}\right)$. We applied a natural 415 logarithmic transformation to linearise the function in Equation 1 and added an interaction 416 term to test our hypothesis that the allometric slope will change with increasing temperature:

$417 \quad \ln N=\ln a_{N}+b_{N} \ln M+E_{N} T_{a r r}+c_{N} \ln M T_{a r r}$,

We analysed the data for all 13 streams with generalised least squares models and 419 linear mixed effects models, using the 'gls' and 'lme' functions in the 'nlme' package of $\mathrm{R}$ 420 3.2.0, with 'ImeControl' parameters specified to deal with convergence issues (see R code in 421 Supplementary Methods). Species identity was included as a random factor, to account for 422 differences in community composition between streams ${ }^{21-23}$. Specifically, we accounted for the possibility that abundance could be different for each species (i.e. a random intercept) and 424 that the effect of body mass and/or temperature on abundance could also be different for each species (i.e. random slopes). We compared models including the full fixed-effect structure 426 plus all possible combinations of the random structure using both Aikake Information Criterion (AIC) and top-down hypothesis testing with the likelihood ratio test ${ }^{44}$. The random structure with species identity influencing $a_{N}, b_{N}$, and $E_{N}$, but not $c_{N}$, was identified as the best model using both approaches $(\Delta \mathrm{AIC}>2.39 ; p=0.009$ in a likelihood ratio test against 430 the next best model). We used this structure in subsequent analyses, set 'method = "ML" ' in 431 the 'Ime' function, and performed AIC comparison and likelihood ratio tests on all possible combinations of the fixed-effect structure ${ }^{44}$. The full model (i.e. Equation 3) was identified as 
433 the best model using both model selection approaches ( $\triangle \mathrm{AIC}>6.57 ; p<0.001$ for the 434 interaction term in a likelihood ratio test).

435 Brown trout occur as the apex predator in a subset of streams ${ }^{22}$ and are orders of 436 magnitude larger than all other species. To rule out the possibility that changes in $M N$-scaling 437 were solely driven by this large predator, we repeated the analysis with this species excluded.

438 We carried out all the same model selection procedures as above. The best-fitting model once 439 again contained the random structure with species identity influencing $a_{N}, b_{N}$, and $E_{N}$, but not $c_{N},(\Delta \mathrm{AIC}>2.36 ; p=0.010$ in a likelihood ratio test against the next best model $)$ and the full

441 fixed-effect structure $(\Delta \mathrm{AIC}>6.42 ; p=0.019$ for the interaction term in a likelihood ratio 442 test). For both analyses, we set 'method = "REML" ' before extracting model summaries and 443 partial residuals from the best-fitting model ${ }^{44}$. Note that the models were always fitted to the 444 raw data collected from the streams, with residuals only extracted for a visual representation 445 of the best-fitting models, excluding the noise explained by the random effect of species 446 identity (see R code in Supplementary Methods).

Trophic group biomass and trophic transfer efficiency

449 To determine the proximate drivers of the observed changes across the temperature gradient in $M N$-scaling, associations with temperature of the total abundance $\left(\mathrm{m}^{-2}\right)$, abundance-weighted mean body mass $(\mathrm{mg})$, and total biomass $\left(\mathrm{mg} \mathrm{m}^{-2}\right)$ of diatoms, 452 invertebrates, and fish were explored with linear regression analysis. We also calculated a 453 predicted metric of trophic transfer efficiency, $T E$, to determine whether the observed changes in $M N$-scaling with temperature altered the energy flow through the system: $\ln T E=\left(b_{1}-b_{0}\right) \ln M R$, ecosystems in which the biota share a common energy source ${ }^{6,45}$, and $M R$ is the consumer- 
resource body mass ratio ${ }^{46} . M R$ was estimated using mean species body mass values and consumer-resource feeding links previously established for the Hengill system ${ }^{22}$. The temperature dependencies of $M R$ and $T E$ were explored with linear regression analysis. Note

461 that all linear regressions in the study were performed according to the equation:

$462 \ln R V=\ln a_{R V}+E_{R V} T_{a r r}$,

463 where $R V$ is the response variable of interest (either $M R, T E$, chlorophyll, or the total 464 abundance, abundance-weighted mean body mass, or total biomass of each trophic group) 465 and all other terms are the same as in Equation 3.

466

\section{Bioenergetic model}

We constructed a bioenergetic population dynamical model to describe the dynamical change of the three main trophic groups in Hengill: diatoms (group 1), invertebrates (group 2), and fish (group 3). These trophic groups form a food chain, and the change of their

471 biomasses through time was modelled as follows:

$472 \quad \frac{d B_{1}}{d t}=r B_{1}\left(1-\frac{B_{1}}{K}\right)-y_{2} B_{1} B_{2}$

$\frac{d B_{2}}{d t}=e_{2} y_{2} B_{1} B_{2}-x_{2} B_{2}-y_{3} B_{2} B_{3}$

$474 \quad \frac{d B_{3}}{d t}=e_{3} y_{3} B_{2} B_{3}-x_{3} B_{3}$

475 Here, $B_{1}, B_{2}$, and $B_{3}$ denote the biomass of diatoms, invertebrates, and fish, respectively (mg $\left.476 \mathrm{~m}^{-2}\right) ; r$ is the maximum mass-specific growth rate of diatoms $\left(\right.$ day $\left.^{-1}\right) ; K$ is the carrying 477 capacity $\left(\mathrm{mg} \mathrm{m}^{-2}\right) ; x_{i}$ is the mass-specific metabolic rate of trophic group $i\left(\right.$ day $\left.^{-1}\right) ; y_{i}$ 478 represents the attack rate of trophic group $i\left(\mathrm{~m}^{2} \mathrm{mg}^{-1} \mathrm{day}^{-1}\right) ; e_{2}=0.45$ is the assimilation 479 efficiency when invertebrates consume diatoms ${ }^{47}$; and $e_{3}=0.85$ is the assimilation efficiency 480 when fish consume invertebrates ${ }^{47}$. In simple terms, this model estimates changes in the 
biomass of: (1) diatoms, as their growth (determined by $r$ and $K$ ) minus their consumption by invertebrates (determined by $y_{2}$ ); (2) invertebrates, as the assimilated proportion of the diatom biomass that they consume (determined by $e_{2}$ and $y_{2}$ ), minus their metabolic demand (determined by $x_{2}$ ), minus their consumption by fish (determined by $y_{3}$ ); and (3) fish, as the assimilated proportion of the invertebrate biomass that they consume (determined by $e_{3}$ and $y_{3}$ ), minus their metabolic demand (determined by $x_{3}$ ). to body mass and temperature as follows:

$r=a_{r} M_{i}^{b_{r}} e^{E_{r} T_{\text {arr }}}, i=1$

490

$$
K=a_{K} M_{i}^{b_{K}} e^{E_{K} T_{a r r}}, i=1,
$$

$x_{i}=a_{x_{i}} M_{i}^{b_{x_{i}}} e^{E_{x_{i}} T_{\text {arr }}}, i=2$ or 3 ,

$$
y_{i}=a_{y_{i}} M_{i}^{b_{y_{i}}} M_{j}^{c_{y_{i}}} e^{E_{y_{i}} T_{\text {arr }}}, i=2 \text { or } 3, j=i-1
$$

493 Here, $a_{r}, a_{K}, a_{x i}$, and $a_{y i}$ are the allometric constants, $b_{r}, b_{K}, b_{x i}$, and $b_{y i}$ are the allometric 494 exponents, and $E_{r}, E_{K}, E_{x i}$, and $E_{y i}$ are the activation energies describing the Arrhenius increase in growth rate, carrying capacity, metabolic rate, and attack rate of trophic group $i$ with temperature, respectively $(\mathrm{eV}) ; c_{y i}$ is the allometric exponent for the resource one trophic 497 level below trophic group $i$; and $M_{i}$ is the mean body mass of trophic group $i(\mathrm{mg})$. We used 498 the abundance-weighted mean trophic group body mass for diatoms $\left(M_{1}=5.8340 \times 10^{-7} \mathrm{mg}\right)$ 499 and fish $\left(M_{3}=9.4854 \times 10^{3} \mathrm{mg}\right)$ because they do not vary systematically with temperature 500 (Fig. S2d,f). The body mass of invertebrates increases with temperature, so we used the 501 following equation to estimate the mean body mass of invertebrates $(\mathrm{mg})$ at each stream 502 temperature:

$503 \ln M_{2}=\ln a_{M_{2}}-E_{M_{2}} T_{a r r}$, 
504 where $E_{M 2}=0.757$ and $\ln a_{M 2}=-2.032$ (Fig. S2e). Note that we carried out a dimensionality 505 reduction to avoid parameter redundancy in the model (see Supplementary Methods and 506 Tables S11 and S12).

\section{Likelihood function}

509 We performed a stability analysis to determine the conditions under which the equilibrium points of the model are stable (see Supplementary Methods). This analysis

511 indicated that for any set of model parameters there was a unique stable equilibrium of the

512 dynamical model for each stream, which provided the model-predicted biomass values for the

513 stream, for those parameters. If $z_{i}^{\text {diatom }}$ is the model-predicted ln-biomass of diatoms and

$514 Z_{i, j}^{\text {diatom }}$ are the three stone scrape measurements of the ln-biomass of diatoms in stream $i$, we

515 assumed the residuals $\varepsilon=z_{i}^{\text {diatom }}-Z_{i, j}^{\text {diatom }}$ should follow a normal distribution with mean 0

516 and standard deviation $\delta_{\text {diatom }}$. These were always finite because diatoms were present in all

517 streams and because all potentially stable equilibria of the dynamical model predicted diatom

518 populations $>0$. The log likelihood for diatoms for all 13 streams was then taken to be:

$\ln L_{\text {all }}^{\text {diatom }}=\sum_{i=1}^{13} \sum_{j=1}^{3}\left(-\frac{1}{2} \ln (2 \pi)-\ln \delta_{\text {diatom }}-\frac{\left(z_{i}^{\text {diatom }}-Z_{i, j}^{\text {diatom }}\right)^{2}}{2 \delta_{\text {diatom }}{ }^{2}}\right)$.

520 We followed an analogous procedure for invertebrates and fish, except we had to 521 accommodate the case in which model-predicted or observed densities were zero. Since 522 invertebrates were observed in all streams, we took the log likelihood for invertebrates to be $523-\infty$ if invertebrates were predicted by the model to be absent from any stream, and otherwise:

$524 \ln L_{\text {all }}^{\text {invert }}=\sum_{i=1}^{13} \sum_{j=1}^{5}\left(-\frac{1}{2} \ln (2 \pi)-\ln \delta_{\text {invert }}-\frac{\left(z_{i}^{\text {invert }}-Z_{i, j}^{\text {invert }}\right)^{2}}{2 \delta_{\text {invert }}{ }^{2}}\right)$,

525 where $z_{i}^{\text {invert }}$ is the model-predicted ln-biomass of invertebrates and $Z_{i, j}^{\text {invert }}$ are the five Surber 
sample measurements of the ln-biomass of invertebrates in stream $i$. For fish, we took the $\log$ likelihood to be $-\infty$ if fish were predicted by the model to be absent from any of the streams in which they were actually observed, or predicted by the model to be present in any of the streams in which they were not observed, and otherwise:

$\ln L_{\text {all }}^{\text {fish }}=\sum_{i \in I}\left(-\frac{1}{2} \ln (2 \pi)-\ln \delta_{\text {fish }}-\frac{\left(z_{i}^{f i s h}-Z_{i}^{f i s h}\right)^{2}}{2 \delta_{\text {fish }}^{2}}\right)$,

531 where $I$ is the set of seven streams in which fish were observed, $z_{i}^{f i s h}$ is the model-predicted

532 ln-biomass of fish, and $Z_{i}^{\text {fish }}$ are the values of fish ln-biomass estimated from three-run depletion electrofishing in stream $i$.

534 Finally, we can get the joint log likelihood for all three groups in the 13 streams:

$\ln L=\ln L_{\text {all }}^{\text {diatom }}+\ln L_{\text {all }}^{\text {invert }}+\ln L_{\text {all }}^{\text {fish }}$

536 This likelihood function corresponds to a statistical model based on sampling log populations

537 from normal distributions centred at equilibrium log population values from the dynamical model, except that when dynamical-model population equilibria are zero, only a sample population estimate of zero is possible. The procedure for dealing with numeric difficulties caused by parameters which yield a value of $-\infty$ is described in Supplementary Methods.

\section{Optimisations}

After dimensionality reduction (see Supplementary Methods), there were 13 parameters

544 to be determined in our model, so we sampled 10,000 different starting parameter 545 combinations from the 13-dimensional hypercube in which each parameter ranged from -100 546 to 100 using a Sobol sequence (with the 'sobolset' function in Matlab 7.12.0). We optimised

547 likelihood for each set and chose the combination of optimised parameters that gave the maximal likelihood (with the 'fminsearchcon' function in Matlab). We then used these values 
549 as the initial point of 2,000,000 iterations in our subsequent Markov Chain Monte Carlo

550 (MCMC) simulations, which were carried out using the Filzbach package in Microsoft Visual

$551 \mathrm{C}++2010$. Filzbach provides a convergence statistic for MCMC chains, with values close to

5521 suggesting mean chain convergence and values $>1.2$ indicating mean non-convergence.

553 The value for our simulations was 1.007. We chose the highest-likelihood parameters ever

554 obtained in this process as the optimised values for each of the 13 parameters in our model

555 (see Table S7). This was entirely a maximum likelihood approach, with MCMC used as an

556 aid to optimisation and as a tool for producing confidence intervals (through profiling), rather

557 than in a hybrid Bayesian fashion. Confidence intervals are those returned by Filzbach. The

558 reason for using both 'fminsearchcon' and MCMC to help optimise was that they have

559 complementary strengths in rapid convergence to a local maximum and broad exploration of 560 the likelihood surface, respectively. Model validation is described in the Supplementary 561 Methods.

562

563 Additional References:

56436 Gudmundsdottir, R. et al. Effects of temperature regime on primary producers in $565 \quad$ Icelandic geothermal streams. Aquatic Botany 95, 278-286 (2011).

56637 Hannesdóttir, E. R., Gíslason, G. M., Ólafsson, J. S., Ólafsson, Ó. P. \& O’Gorman, E.

567 J. Increased stream productivity with warming supports higher trophic levels. $568 \quad$ Advances in Ecological Research 48, 283-340 (2013).

56938 Arnason, B., Theodorsson, P., Björnsson, S. \& Saemundsson, K. Hengill, a high 570 temperature thermal area in Iceland. Bulletin of Volcanology 33, 245-259 (1969).

57139 Abramoff, M. D., Magalhaes, P. J. \& Ram, S. J. Image processing with ImageJ. Biophotonics International 11, 36-42 (2004). 
57340 Sun, J. \& Liu, D. Geometric models for calculating cell biovolume and surface area 574 for phytoplankton. Journal of Plankton Research 25, 1331-1346 (2003).

57541 Rocha, O. \& Duncan, A. The relationship between cell carbon and cell volume in 576 freshwater algal species used in zooplanktonic studies. Journal of Plankton Research $577 \quad 7,279-294(1985)$.

57842 Sicko-Goad, L. M., Schelske, C. L. \& Stoermer, E. F. Estimation of intracellular 579 carbon and silica content of diatoms from natural assemblages using morphometric $580 \quad$ techniques. Limnology and Oceanography 29, 1170-1178 (1984).

$58143 \quad$ Seber, G. A. F. \& Le Cren, E. D. Estimating population parameters from catches large 582 relative to the population. Journal of Animal Ecology 36, 631-643 (1967).

58344 Zuur, A. F., Ieno, E. N., Walker, N. J., Saveliev, A. A. \& Smith, G. M. in Mixed $584 \quad$ effects models and extensions in ecology with $R \quad 101-142$ (Springer, 2009).

58545 Damuth, J. Population-density and body size in mammals. Nature 290, 699-700 $586 \quad(1981)$.

$58746 \quad$ Jennings, S. \& Mackinson, S. Abundance-body mass relationships in size-structured 588 food webs. Ecology Letters 6, 971-974 (2003).

58947 Yodzis, P. \& Innes, S. Body size and consumer-resource dynamics. American $590 \quad$ Naturalist, 1151-1175 (1992).

$59148 \quad$ Vasseur, D. A. \& McCann, K. S. A mechanistic approach for modeling temperaturedependent consumer-resource dynamics. American Naturalist 166, 184-198 (2005). 